VOL. II (1974), I1-14.

\title{
The determination of Fibonacci groups
}

\section{A.M. Brunner}

Fibonacci groups are the groups

$$
\begin{array}{r}
F(2, r)=\operatorname{gp}\left(a_{1}, a_{2}, \ldots, a_{r+2}: a_{i+2}=a_{i+1} a_{i} ; i=1, \ldots, r\right. \\
\left.a_{r+1}=a_{1}, a_{r+2}=a_{2}\right),
\end{array}
$$

where $r$ is a natural number. The groups $F(2,8)$ and $F(2,10)$ are shown to be infinite, thus leaving $F(2,9)$ as the only Fibonacci group whose finiteness or infiniteness has not been determined.

\section{Introduction}

Fibonacci groups are the groups

(1) $F(2, r)=\operatorname{gp}\left(a_{1}, a_{2}, \ldots, a_{r+2}: a_{i+2}=a_{i+1} a_{i} ; i=1, \ldots, r\right.$;

$$
\left.a_{r+1}=a_{1}, a_{r+2}=a_{2}\right)
$$

where $r$ is a natural number.

According to [1] the finite groups $F(2, r)$ are known, except when $r=8,9$ and 10 . This note will establish that $F(2,8)$ and $F(2,10)$ are infinite.

The results now known are as follows: $F(2,0)$ is the free group of rank $2 ; F(2,1)$ and $F(2,2)$ are the trivial group; $F(2,3)$ is the

Received 25 February 1974. The author thanks Professor B.H. Neumann, who first introduced him to Fibonacci groups, for his continued interest and encouragement, and Dr R.M. Bryant for having brought the paper by Johnson, Wamsley and Wright to his attention. 
quaternion group of order $8 ; F(2,4)$ is cyclic of order $5 ; F(2,5)$ is cyclic of order $11 ; F(2,6)$ is the infinite metabelian group $\operatorname{gp}\left(a, b ; b^{-1} a^{2} b=a^{-2}, a^{-1} b^{2} a=b^{-2}\right) ; F(2,7)$ is cyclic of order 29 (established using an electronic computer by John Cannon (1971) and confirmed by Jane Watson); $F(2,8)$ and $F(2,10)$ are infinite; and, when $n \geq 11$ the groups $F(2, n)$ are infinite (Lyndon; see [1]).

In [1] Problem 4 asks whether the factor group of $F(2,10)$ obtained by adding the relation $a_{6}=a_{1}$ to the relations in ( 1 ) is finite. The group turns out to be $\operatorname{gp}\left(a, b ; a^{11}=b^{23}=a^{-4} b^{-1} a b=1\right)$ and has order 11.23. A proof is not given here as it is long, and an easy method for showing $E(2,10)$ to be infinite is given below.

\section{The group $F(2,8)$}

THEOREM 2.1. The group $F(2,8)$ is infirite: an infinite epimorph is the group $\operatorname{sp}\left(c, d ; c^{2}=d^{5}=(c d)^{5}=\left(c d^{2}\right)^{5}=1\right)$.

The proof of the theorem follows from Lemmas 2.2 and 2.3 below.

LEMMA 2.2. The group $H=g p\left(c, d ; c^{2}=d^{5}=(c d)^{5}=\left(c d^{2}\right)^{5}=1\right)$ is an epimorph of $F(2,8)$.

Proof. Firstly the elements $d$ and $c d^{3} c$ generate $H$; for $\left(c d^{3} c\right)^{2}=c d^{3} c^{2} d^{3} c=c d c$ as $c^{2}=d^{5}=1$, and since $(c d)^{5}=1$, the element $c$ is obtained as $c^{-1}=$ dededcded $=d\left(c d^{3} c\right)^{2} d\left(c d^{3} c\right)^{2} d$.

Now set $x_{1}=d, x_{2}=c d^{3} c$ and $x_{n+2}=x_{n+1} x_{n}$ for $n=1,2, \ldots$. An easy calculation using the relations of $H$ shows that $x_{3}=c d^{3} c d$, $x_{4}=c d^{3} c d c d^{3} c, x_{5}=c d^{2} c d^{-1} c, x_{6}=c d^{2} c d^{2} c d c d^{3} c, x_{7}=c d^{2} c d^{2}$, $x_{8}=d^{-1} c d^{3} c, x_{9}=d$ and $x_{10}=c d^{3} c$.

The required epimorphism is the one mapping $a_{i}$ to $x_{i}$ for $i=1,2, \ldots, 10$.

LEMMA 2.3. The group $H=\operatorname{gp}\left(c, d ; c^{2}=d^{5}=(c d)^{5}=\left(c d^{2}\right)^{5}=1\right)$ is 
infinite.

Proof. The group

$$
\begin{aligned}
A=\operatorname{gp}\left(c_{0}, c_{1}, c_{2}, c_{3} ; c_{0}^{2}=c_{1}^{2}=c_{2}^{2}=\right. & c_{3}^{2}=1, \\
& \left.\quad c_{0} c_{1} c_{2} c_{3}=c_{1} c_{3} c_{0} c_{2},\left(c_{0} c_{1} c_{2} c_{3}\right)^{2}=1\right)
\end{aligned}
$$

is infinite; indeed, the free product of two cyclic groups of order 2 is an epimorph, as can easily be seen by placing $c_{0}=1$ and $c_{2}=c_{3}$. Moreover $A$ has an automorphism $X$ of order 5 , with $c_{i} X=c_{i+1}$ $(i=0,1,2)$ and $c_{3} X=c_{0} c_{1} c_{2} c_{3}$.

Since $A$ is infinite, an extension

$$
\begin{aligned}
\operatorname{gp}\left(c_{0}, c_{1}, c_{2}, c_{3}, d: d^{5}=c_{0}^{2}=1 ; c_{i}^{d}\right. & =c_{i+1}, i=0,1,2 ; \\
& \left.c_{3}^{d}=c_{0} c_{1} c_{2} c_{3}, c_{0} c_{1} c_{2} c_{3}=c_{1} c_{3} c_{0} c_{2}\right),
\end{aligned}
$$

of $A$ by means of $X$ is infinite also. Rewritten in terms of its generators $c=c_{0}, d$ this becomes

$$
\operatorname{gp}\left(c, d ; c^{2}=d^{5}=1, c^{d^{4}}=c c^{d} c^{d^{2}} c^{d^{3}}=c^{d} c^{d^{3}} c c^{d^{2}}\right)
$$

or,

$$
\operatorname{gp}\left(c, d ; c^{2}=d^{5}=\left(c d^{-1}\right)^{5}=\left(c d^{-2}\right)^{5}=1\right)
$$

which is $H$.

\section{The group $F(2,10)$}

THEOREM 3.1. The group $F(2,10)$ is infinite.

Proof. Let

$$
A_{1}=\left[\begin{array}{rrr}
1 & 1 & 0 \\
-1 & 0 & 0 \\
0 & 0 & 1
\end{array}\right], \quad A_{2}=\left[\begin{array}{lll}
0 & 1 & 0 \\
0 & 0 & 1 \\
1 & 0 & 0
\end{array}\right]
$$

be matrices over the integers, and set $A_{n+2}=A_{n+1} A_{n}$ for $n=1,2, \ldots$. A short calculation shows that $A_{11}=A_{1}$ and $A_{12}=A_{2}$, so there is an 
epimorphism of $F(2,10)$ onto the matrix group generated by $A_{1}$ and $A_{2}$ mapping $a_{i}$ to $A_{i}$ for $i=1,2, \ldots, 12$.

Now

$$
A_{7}=A_{1}^{-2} A_{2} A_{1}^{-1} A_{2}=\left[\begin{array}{rrr}
-1 & -1 & -1 \\
0 & 1 & 1 \\
0 & 0 & -1
\end{array}\right]
$$

so that

$$
A_{7}^{2}=\left[\begin{array}{lll}
1 & 0 & 1 \\
0 & 1 & 0 \\
0 & 0 & 1
\end{array}\right] \text {, }
$$

an element which clearly has infinite order. It follows that $F(2,10)$ is infinite.

\section{Reference}

[1] D.L. Johnson, J.W. Wamsley and D. Wright, "The Fibonacci groups", $J$. London Math. Soc. (to appear).

Department of Mathematics,

Monash University,

Clayton,

victoria.

Present address:

Department of Mathematics,

University of Western Australia,

Nedlands,

Western Australia. 